\title{
Distributed DC Optimal Power Flow for Radial Networks Through Partial Primal Dual Algorithm
}

\author{
Vahid Rasouli Disfani, Student Member, IEEE, Lingling Fan, Senior Member, IEEE, Zhixin Miao, Senior Member, \\ IEEE
}

\begin{abstract}
In a smart grid platform, where more distributed resources are joining everyday and each of them wills to keep its own privacy and shares as less information as possible with the grid management, there is a motivation for transition from the current centralized structures to more distributed ones. In this paper, a distributed method is presented to solve DC Optimal Power Flow (DC-OPF) for radial networks based on partial Primal-Dual algorithm. The algorithm development process starts from an economic dispatch (ED) problem, derives a distributed ED, and finally makes a bridge from the distributed ED formulation to a distributed algorithm for DC-OPF. It is proved in the paper that the optimal solution of the distributed algorithm complies with the necessary and satisfactory KKT conditions of DC-OPF problem. The algorithm is tested on a radial power network with and without network congestion, where the results converge to the optimal solution.
\end{abstract}

\section{INTRODUCTION}

$\mathbf{S}$ IGNIFICANT increase in amount of private distributed energy resources such as renewable energy resources, energy storage systems, distributed generations, and priceresponsive demands is expected in the future smart grid. Not only will it lead to a competitive environment in power system, but also it will introduce a huge volume of control variables in the entire power network. Further, private agents are generally not willing to reveal their own control variables and economical data due to either privacy or economical reasons. These two facts have motivated the recent interest in distributed methods in power systems.

Optimal power flow (OPF, AC-OPF), which determines the most economic schedule to operate generation units and controllable demands in a power network, has always been a challenging problem in power system operation due to its inherent nonlinearity and nonconvexity. Several works are reported in the literature to tackle the OPF problem in a distributed fashion [1]-[5]. All of these algorithms separate the entire network into several sub-networks, each of which solves its own OPF problem at each iteration mostly using interior point method. These algorithms then develop an information flow scheme and an update process to push the solution toward the optimal solution. In [1], coarse-grained distributed implementation is used to parallelize the OPF problem, which is extended in [2] and compared to some other distributed approaches. Alternative-Direction Method of Multipliers (ADMM) is employed in [3] to develop distributed OPF solution. The same method (ADMM) is used in [4]

V.R. Disfani, L. Fan and Z. Miao are with the Department of Electrical Engineering, University of South Florida, Tampa, FL 33620 (Email: linglingfan@usf.edu). to determine a distributed method for semi-definite programbased (SDP-based) formulation of OPF problem, which is first proposed in [6]. A modified subgradient-based method along with Lower-Upper-Bound-Switching (LUBS) method is presented in [5] for distributed AC-OPF problem. In the LUBS method, the microgrids update their desired prices based of which the main grid iteratively defines its optimal power export/import level from each microgrid. The main grid also compares the upperbound and lowerbound of the optimal solution to guarantee zero duality gap of the final solution.

Several distributed algorithms have been presented for economic dispatch and DC-OPF problems. A gradient-based approach along with consensus-based update process using center-free algorithms [7] is employed in [8] to develop a distributed platform for online optimal generation control or economic dispatch. In fact, the gradient-based method guarantees the power balance in the entire network using local frequency signal while the consensus-based process unifies all the locational marginal prices (LMP) on their unique final values. The center-free algorithms have fast convergence speed but are not appropriate for DC-OPF problems because they cannot address the difference between nodal prices due to line constraints. Thus, a subgradient method is employed to develop the consensus-based update process for distributed DC-OPF problems in [9]-[11]. The idea of separating the entire network into some sub-networks, which is widely used to develop distributed AC-OPF solutions, is also practiced for distributed DC-OPF problems in the aforementioned references. At each iteration, each sub-network (agent) solves its own DC-OPF problem locally; then, a pricing mechanism employing subgradient method updates the price using the information gathered from the neighbors, and iterations continues until convergence is met. The most distributed way to define these sub-networks is to consider each bus of the system as a distinct agent, which is proposed in [12]. In this algorithm, which is developed based on subgradient method, each bus defines its optimal power generation level and updates its own multipliers, shares some data with its neighbors, and continues to iterate until global convergence is reached.

In this paper, a distributed algorithm considering each bus as a distinct agent is proposed to solve DC-OPF problem for radial networks. The algorithm adopted in this paper is partial primal-dual algorithm, which guarantees the convergence rate toward globally optimal solution for uniformly convex problems [13] and has several advantageous features for radial networks. Compared to dual decomposition based algorithms, where sub-optimization problems are solved given Lagrange multipliers, in a primal-dual algorithm, there is no optimization 
carried out for sub-problems. Instead, an updating procedure is adopted to update the control variables. Compared to centerfree algorithm, the update process in the proposed algorithm has physical meaning of price updating and is able to address the nodal price differences due to network constraints. It is also faster than the algorithm proposed in [12] due to less volume of updating processes, especially on the Lagrange multipliers of inequality constraints.

The partial primal-dual algorithm has been employed in [14], [15] to explain that the droop control is aligned with a primal-dual process. Our development process starts from economic dispatch problem and applies dual decomposition techniques to extract a distributed economic dispatch. After applying primal decomposition technique to the distributed economic dispatch problem, we further develop a distributed DC-OPF algorithm. The solution sought by the algorithm is proved to be optimal since it satisfies the necessary and satisfactory KKT conditions of the DC-OPF problem for radial networks. In order to demonstrate its efficiency, the algorithm is tested on a radial power network (a modified version of IEEE 9-bus test system).

The rest of the paper is organized as follows. Section II explains the DC-OPF problem formulation and KKT necessary conditions. The distributed DC-OPF algorithm is described in Section III. Section IV provides the simulation results. Section $\mathrm{V}$ concludes the paper.

\section{DC-OPF AND KKT CONDITIONS}

Consider a power network containing several generators and controllable loads. The network has finite numbers of buses belonging to $\mathcal{N}$ and finite numbers of branches (edges) belonging to $\mathcal{E}$. The numbers of nodes and branches are then $|\mathcal{N}|$ and $|\mathcal{E}|$ respectively. Generation, demand, and voltage angle on bus $i \in \mathcal{N}$ are denoted by $P_{g i}, P_{d i}$, and $\theta_{i}$, whereas the power flow on line $(i, j) \in \mathcal{E}$ from node $i$ to $j$ is called $P_{i j}$.

A typical formulation of DC Optimal Power Flow is presented below.

a) $D C-O P F$ :

$$
\begin{aligned}
& \text { maximize } W\left(\mathbf{P}_{\mathbf{g}}, \mathbf{P}_{\mathbf{d}}\right)=\sum_{i \in \mathcal{N}} U_{i}\left(P_{d i}\right)-C_{i}\left(P_{g i}\right) \\
& \text { over: } \mathbf{P}_{\mathrm{g}}, \mathbf{P}_{\mathbf{d}}, \mathbf{P}_{\mathbf{L}}, \theta \\
& \text { subject to: } \sum_{i \in \mathcal{N}} P_{g i}=\sum_{i \in \mathcal{N}} P_{d i} \\
& P_{g i}-P_{d i}=\sum_{j:(i, j) \in \mathcal{E}} P_{i j} \\
& \underline{P_{g i}} \leq P_{g i} \leq \overline{P_{g i}} \quad \forall i \in \mathcal{N} \\
& \underline{P_{d i}} \leq P_{d i} \leq \overline{P_{d i}} \quad \forall i \in \mathcal{N} \\
& P_{i j}+P_{j i}=0 \quad \forall(i, j) \in \mathcal{E} \\
& -\overline{P_{i j}} \leq P_{i j} \leq \overline{P_{i j}} \quad \forall(i, j) \in \mathcal{E} \\
& P_{i, j}=\frac{\theta_{i}-\theta_{j}}{x_{i j}} \quad \forall(i, j) \in \mathcal{E} \\
& \theta_{0}=0
\end{aligned}
$$

where $\mathbf{P}_{\mathbf{g}}=\left\{P_{g i}: i \in \mathcal{N}\right\}, \mathbf{P}_{\mathbf{d}}=\left\{P_{d i}: i \in \mathcal{N}\right\}$, $\mathbf{P}_{\mathbf{L}}=\left\{P_{i j}: i \in \mathcal{E}\right\}$, and $\theta=\left\{\theta_{i}: i \in \mathcal{N}\right\}$. Besides, (1a) describes the objective function so as to maximize the welfare in the entire network, (1b)- (1c) denote total and nodal power balance, and (1d)-(1e) explain the inequality constraints on power generation and demand. (1f) guarantees the network to be lossless, and line capacity limits are also explained by $(1 \mathrm{~g})$. (1h) describes the line power in terms of the voltage angles of the buses across each line. (1i) fixes the voltage angle of the slack bus on zero.

Remark 1: Although the constraint (1b) holds if the constraints (1c) and (1f) satisfied, it is mentioned here to make emphasis of total power balance. Likewise, (1f) is mentioned to be emphasized though it is explicitly guaranteed by the constraint (1h).

Remark 2: As the equality constraints are affine functions and the inequality constraints are continuously differentiable convex functions in the optimization problem (1), the feasible solution set is a convex set.

Condition 1: Assuming that the generation cost function $C_{i}\left(P_{g i}\right)$ and the demand utility function $U_{i}\left(P_{d i}\right)$ are respectively strictly convex and strictly concave for any $i \in \mathcal{N}$, the welfare function is strictly concave. Therefore, the problem (1) is a convex optimization problem.

Remark 3: If DC-OPF is defined over a radial power network, any vector $P_{L}$ can be mapped on a unique vector $\theta$ where $\theta_{0}=0$. Therefore, removing the constraints (1h)-(1i) does not affect the optimal solution of the DC-OPF problem described in (1) for a radial power network, so the constraints (1h)-(1i) are neglected in the DC-OPF problem, hereafter.

According to Karush-Kuhn-Tucker (KKT) conditions, if the solution $\left(\mathbf{P}_{\mathbf{g}}^{*}, \mathbf{P}_{\mathbf{d}}^{*}, \mathbf{P}_{\mathbf{L}}^{*}\right)$ is a local optimum of (1), it necessarily satisfies the following regulatory conditions,

Stationary:

$$
\begin{aligned}
& \sum_{i \in \mathcal{N}}\left\{\frac{\partial C_{i}}{\partial P_{g i}}\left(P_{g i}^{*}\right)-\left(\lambda_{i}-\overline{\mu_{g i}}+\underline{\mu_{g i}}\right)\right\}=0 \\
& \sum_{i \in \mathcal{N}}\left\{\frac{\partial U_{i}}{\partial P_{d i}}\left(P_{d i}^{*}\right)-\left(\lambda_{i}+\overline{\mu_{d i}}-\underline{\mu_{d i}}\right)\right\}=0
\end{aligned}
$$

Complementary slackness:

$$
\begin{array}{ll}
\overline{\mu_{g i}}\left(\overline{P_{g i}}-P_{g i}\right)=\underline{\mu_{g i}}\left(P_{g i}-\underline{P_{g i}}\right)=0 & \forall i \in \mathcal{N} \\
\overline{\mu_{d i}}\left(\overline{P_{d i}}-P_{d i}\right)=\underline{\mu_{d i}}\left(P_{d i}-\underline{P_{d i}}\right)=0 & \forall i \in \mathcal{N}
\end{array}
$$

Dual feasibility:

$$
\min \left\{\overline{\mu_{g i}}, \underline{\mu_{g i}}, \overline{\mu_{d i}}, \underline{\mu_{d i}}\right\} \geq 0 \quad \forall i \in \mathcal{N}
$$

Primal feasibility:

$$
\text { (1c) }-(1 \mathrm{~g})
$$

where the vector $\lambda \in \mathbb{R}^{|\mathcal{N}|}$ denotes Lagrange multipliers corresponding to the constraint (1c), and the vectors $\underline{\mu} \in \mathbb{R}^{|\mathcal{N}|}$ and $\bar{\mu} \in \mathbb{R}^{|\mathcal{N}|}$ are Lagrange multipliers of the inequality constraints (1d)-(1e).

Due to the convexity of the original problem (1), the KKT necessary conditions described in (2) are also sufficient for optimality of $\left(\mathbf{P}_{\mathbf{g}}^{*}, \mathbf{P}_{\mathbf{d}}^{*}, \mathbf{P}_{\mathbf{L}}^{*}\right)$. 


\section{Design OF Distributed DC-OPF}

Consider a new optimization problem which is defined same as the optimization problem (1) if the constraints corresponding to the lines i.e. (1c),(1f)-(1i) are neglected. The new optimization problem is exactly the problem of Economic Dispatch as defined below.

Economic Dispatch (ED):

$$
\begin{array}{rlr}
\text { minimize } & \sum_{i \in \mathcal{N}} C_{i}\left(P_{g i}\right)-U_{i}\left(P_{d i}\right) & \\
\text { over: } & \mathbf{P}_{\mathbf{g}}, \mathbf{P}_{\mathbf{d}} & \\
\text { subject to: } & \sum_{i \in \mathcal{N}} P_{g i}=\sum_{i \in \mathcal{N}} P_{d i} & \\
& \underline{P_{g i}} \leq P_{g i} \leq \overline{P_{g i}} & \forall i \in \mathcal{N} \\
& \underline{P_{d i}} \leq P_{d i} \leq \overline{\mathcal{N}}
\end{array}
$$

Note that (3b) does not require the nodal power balance of generation and demand, but only balance across the entire network. If the Condition 1 holds for the objective function (3a), the optimization problem (3) is also convex.

The dual representation of (3) is

$$
\max _{\lambda} \sum_{i \in \mathcal{N}} \underbrace{\left\{\begin{array}{c}
\min _{P_{g i}, P_{d i}} C_{i}\left(P_{g i}\right)-U_{i}\left(P_{d i}\right)+\lambda\left(P_{d i}-P_{g i}\right) \\
\text { s.t. } \left.\begin{array}{c}
P_{g i} \leq P_{g i} \leq \overline{P_{g i}} \\
\overline{P_{d i}} \leq P_{d i} \leq \overline{P_{d i}}
\end{array}\right\}
\end{array}\right.}_{\Phi_{i}^{\dagger}(\lambda)}
$$

where the optimal solution of the minimization problem can be explicitly defined as

$$
\Phi_{i}^{\dagger}(\lambda):=C_{i}\left(P_{g i}^{\dagger}\right)-U_{i}\left(P_{d i}^{\dagger}\right)
$$

with

$$
\begin{aligned}
P_{g i}^{\dagger}(\lambda) & :=\left[M C_{i}^{-1}(\lambda)\right]^{\overline{P_{g i}}} \\
P_{d i}^{\dagger}(\lambda) & :=\left[M U_{i}^{-1}(\lambda)\right]_{\underline{P_{d i}}}^{\overline{P_{d i}}}
\end{aligned}
$$

where $M C_{i}\left(P_{g i}\right)=\frac{\partial C_{i}}{\partial P_{g i}}$ and $M U_{i}\left(P_{d i}\right)=\frac{\partial U_{i}}{\partial P_{d i}}$ are the marginal cost and marginal utility corresponding to bus $i$, and $[\cdot]_{a}^{b}$ denotes $\max \{\min \{\cdot, b\}, a\}$ for $a, b \in \mathbb{R}, a \leq b$.

The objective function $\Phi_{i}^{\dagger}(\lambda)$ is not separable across buses $i \in \mathcal{N}$ since it has a scalar variable $\lambda$ which is common between all $i \in \mathcal{N}$. The following distributed version of the dual problem is proposed in this paper by introducing the vector $\lambda:=\left(\lambda_{i}, i \in \mathcal{N}\right)$. The variables $\lambda_{i}$ are constrained to be equal for all $i \in \mathcal{N}$ at optimality i.e. $\lambda_{i}=\lambda_{j} \forall i, j \in \mathcal{N}$.

\section{A. Distributed Economic Dispatch}

\section{Distributed Economic Dispatch (DED):}

$$
\begin{aligned}
\max _{\lambda} & \Phi(\lambda):=\sum_{i \in \mathcal{N}} \Phi_{i}^{\dagger}\left(\lambda_{i}\right) \\
\text { s.t. } & \lambda_{i}=\lambda_{j} \quad \forall(i, j) \in \mathcal{E}
\end{aligned}
$$

Since the power network is assumed to be a connected grid, it is enough to define the constraints ( $7 b$ ) across the lines.
The following two results suggest solving the distributed optimization problem (7) rather than ED. The unique optimal point $\left(P_{g}^{*}, P_{d}^{*}\right)$ will be then recovered from the unique dual optimal $\lambda^{*}$. A proof has been provided in Appendix B of [15] for these two results.

Lemma 1: The objective function $\Phi$ of DED is strictly concave over $\mathbb{R}^{|\mathcal{N}|}$.

Lemma 2:

i- DED has a unique optimal point $\left(\lambda^{*}\right)$ with $\lambda_{i}^{*}=\lambda_{j}^{*}=$ $\lambda^{*}$ for all $(i, j) \in \mathcal{E}$

ii- ED has a unique optimal point $\left(P_{g}^{*}, P_{d}^{*}\right)$ where $P_{g i}^{*}=$ $P_{g i}^{\dagger}\left(\lambda^{*}\right)$ and $P_{d i}^{*}=P_{d i}^{\dagger}\left(\lambda^{*}\right)$.

Applying the Lagrange relaxation upon the equality constraints leads to the following Lagrange function of DED which will be employed to derive a distributed solution.

$$
L(\lambda, \pi):=\sum_{i \in \mathcal{N}} \Phi_{i}^{\dagger}\left(\lambda_{i}\right)-\sum_{(i, j) \in \mathcal{E}} \pi_{i j}\left(\lambda_{j}-\lambda_{i}\right)
$$

where $\pi \in \mathbb{R}^{|\mathcal{E}|}$ is the vector of lagrange multipliers for the equality constraints (7b). In fact, any multiplier $\pi_{i j}$ measures the cost of mismatch between $\lambda_{i}$ and $\lambda_{j}$ across the line $(i, j) \in$ $\mathcal{E}$. A partial primal-dual algorithm for DED takes the form below for (7) and (8):

$$
\begin{aligned}
& \lambda_{i}(k+1)=\lambda_{i}(k)+\gamma_{i} \frac{\partial L}{\partial \lambda_{i}}(\lambda(k), \pi(k))=\lambda_{i}(k)+ \\
& \gamma_{i}\left(P_{d i}^{\dagger}\left(\lambda_{i}(k)\right)-P_{g i}^{\dagger}\left(\lambda_{i}(k)\right)+\sum_{j:(i, j) \in \mathcal{E}} \pi_{i j}(k)\right) \\
& \pi_{i j}(k+1)=\pi_{i j}(k)-\xi_{i j} \frac{\partial L}{\partial \theta_{i j}}(\lambda(k+1), \pi(k)) \\
& =\pi_{i j}(k)+\xi_{i j}\left(\lambda_{j}(k+1)-\lambda_{i}(k+1)\right)
\end{aligned}
$$

where $\gamma_{i}$ and $\xi_{i j}$ are positive real numbers.

\section{B. Distributed DC Optimal Power Flow}

It is implied from the stationary point of (9) that $P_{d i}^{*}-$ $P_{g i}^{*}-\sum_{j:(i, j) \in \mathcal{E}} \pi_{i j}^{*}=0$ for any $i \in \mathcal{N}$, which is identical to the constraint (1c) if the $\pi$ is identified by $\mathbf{P}_{\mathbf{L}}$. Therefore, the DC-OPF problem (1) can be recovered from distributed economic dispatch by adding the following two constraints:

$$
\begin{array}{ll}
\pi_{i j}+\pi_{j i}=0 & \forall(i, j) \in \mathcal{E} \\
-\overline{P_{i j}} \leq \pi_{i j} \leq \overline{P_{i j}} & \forall(i, j) \in \mathcal{E}
\end{array}
$$

where (11) and (12) are identical to the constraints (1f) and (1g), respectively. With these definitions, the parameters $\lambda_{i}$ and $\pi_{i j}$ have the physical meanings of locational marginal price on bus $i \in \mathcal{N}$ and power flow on line $(i, j) \in \mathcal{E}$, which exclude any information of the agents' cost functions and maintain their privacy.

Although the agents are required to follow specific instructions securing convergence, there is no need for them to adopt same updating rules with same coefficients in a cooperative algorithm. However, some parameters must be meaningful before being fed back into the algorithm at the next iteration. For example, the constraint (11) is automatically satisfied if $\pi_{i j}=-\pi_{j i}$ for any $(i, j) \in \mathcal{E}$ at initial point 
and the corresponding update coefficients are equal in the entire process i.e. $\xi_{i j}=\xi_{j i}$ for all $(i, j) \in \mathcal{E}$. Since it is not guaranteed by the agent to start from an ideal $\pi$ or to keep their own coefficients exactly equal to the neighbors' parameters, the solution proposed in this paper to secure the constraint (11) is applying a center-free-based update over $\pi_{i j}$ and $-\pi_{j i}$ at each iteration i.e.

$$
\pi_{i j}=\frac{\pi_{i j}-\pi_{j i}}{2} \forall(i, j) \in \mathcal{E}
$$

which are carried out by the agent $i$ after receiving $\xi_{j i}$ from its neighboring agents $j:(i, j) \in \mathcal{E}$. The center-free-based update method causes a coefficient equal to $\left(\xi_{i j}+\xi_{j i}\right) / 2$ to be applied to update both $\pi_{i j}$ and $\pi_{j i}$ at each iteration before updating $\lambda$.

Replacing (10) which describes the update process of $\pi$ with the equation below would also satisfy (12).

$$
\pi_{i j}(k+1)=\left[\pi_{i j}(k)+\theta_{i j}\left(\lambda_{j}-\lambda_{i}\right)\right]_{-\overline{P_{i j}}}^{\overline{P_{i j}}}
$$

Remark 4: All the necessary and sufficient conditions for the optimality of $\left(P_{g}^{*}, P_{d}^{*}, P_{L}^{*}\right)$ described in (2a)-(2f) are satisfied by the proposed partial primal-dual algorithm. The conditions (2a)-(2b) as well as the constraints (1d)-(1e) are satisfied by (5)-(6) with the definitions below,

$$
\begin{aligned}
\underline{\mu_{g i}} & :=\max \left\{0, M C^{-1}\left(P_{g i}\right)-\lambda_{i}\right\} \\
\overline{\mu_{g i}} & :=\max \left\{0, \lambda_{i}-M C^{-1}\left(P_{g i}\right)\right\} \\
\underline{\mu_{d i}} & :=\max \left\{0, M U^{-1}\left(P_{d i}\right)-\lambda_{i}\right\} \\
\overline{\mu_{d i}} & :=\max \left\{0, \lambda_{i}-M U^{-1}\left(P_{d i}\right)\right\}
\end{aligned}
$$

There is no need to change the communication network to tackle the generation/demand capacity constraints in this paper although some reconfigurations are introduced in [8] as required actions for such a purpose. The dual feasibility and complementary slackness conditions (2c)-(2e) are also satisfied by (15)-(18). The equality constraints (1c) and (1f) are satisfied by the stationary points of (9) and (13), respectively.

To summarize, the partial primal-dual algorithm described by (9) and (13)-(14) solves the optimization below, which is called Distributed DC-OPF due to its distributed properties.

\section{Distributed DC-OPF:}

$$
\begin{array}{ll}
\min _{\pi} & \max _{\lambda} \sum_{i \in \mathcal{N}}\left(\Phi_{i}^{\dagger}\left(\lambda_{i}\right)-\sum_{j:(i, j) \in \mathcal{E}} \pi_{i j}\left(\lambda_{j}-\lambda_{i}\right)\right) \\
\text { s.t. } & -\overline{P_{i j}} \leq \pi_{i j}=-\pi_{j i} \leq \overline{P_{i j}}
\end{array}
$$

Algorithm 1 illustrates a distributed algorithm to solve the DDC-OPF problem (19) through an iterative process.

\section{Simulation Results}

In order to demonstrate the efficiency of the algorithm proposed in the paper, it is tested against a modified version of IEEE 9-bus test system, including three generators on buses $1-3$. This test system is a meshed network including only one loop. In this study, the line between buses 4 and 9 is removed to create a radial test system. The details of the cost functions and limits of generation units are provided in Table I.

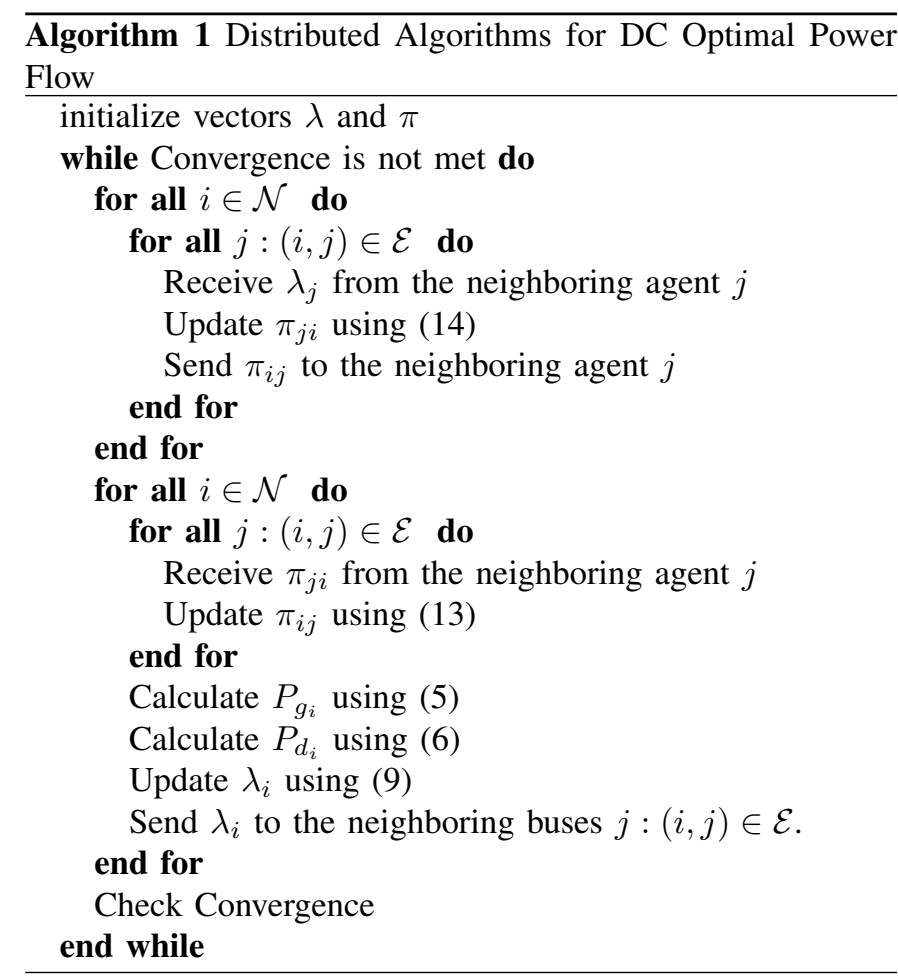

TABLE I

DETAILS OF GENERATORS IN IEEE 9-BUS TEST SYSTEM

\begin{tabular}{|c|c|c|c|c|c|c|}
\multicolumn{7}{c|}{$C\left(P_{g}\right)=a P_{g}^{2}+b P_{g}+c$} \\
\hline Gen \# & Bus \# & $P_{g}$ & $P_{g}$ & $a$ & $b$ & $c$ \\
\hline 1 & 1 & 10 & 50 & 0.11 & 5 & 150 \\
\hline 2 & 2 & 10 & 300 & .085 & 1.2 & 600 \\
\hline 3 & 3 & 10 & 270 & .1225 & 1 & 335 \\
\hline
\end{tabular}

The coefficients used for updating process are selected as $\gamma_{i}$ for any $i \in \mathcal{N}$ and $\xi_{i j}=8$ for any $(i, j) \in \mathcal{E}$. The algorithm starts the iteration from the initial values of $\lambda_{i}=0$ for any $i \in \mathcal{N}$ and $\pi_{i j}=0$ for any $(i, j) \in \mathcal{E}$.

\section{A. Case 1: Without line congestion}

This case is studied against the modified IEEE 9-bus test system with the above-mentioned modifications. Fig. 1 depicts the simulation results corresponding to this case study. The algorithm converges to the optimal solution, which is benchmarked against the results obtained by MATPOWER 4.1 [16], in 200 iterations. As none of the network lines is congested in this case, all locational marginal prices $\lambda$ converge to a unique price $\left(\lambda_{i}=27.71 \frac{\$}{\mathrm{MWhr}} \forall i \in \mathcal{N}\right)$, as illustrated in Fig. 1-a. According to Fig. 1-b, The generator 1 provides its maximum power output $\left(P_{g 1}=50 \mathrm{MW}\right)$ to the system while the other two generators are operated at $P_{g 2}=156 \mathrm{MW}$ and $P_{g 3}=109$ MW. Total power generation supplies the total power demand with a cost equal to $\$ 5430.18$. The power flow on the lines are also provided in Fig. 1-c, where the line power $P_{i j}$ and $P_{j i}$ are shown by solid and dashed lines but in the same color.

\section{B. Case 2: With line congestion}

In order to investigate the algorithm in congested networks, the maximum power flow on the line between buses 7 and 
$8\left(L_{78}\right)$ is assumed to be $10 \mathrm{MW}$. The algorithm proposed converges to the same solution as MATPOWER suggests in 300 iterations. Fig. 2 illustrates the simulation results for this case study. Unlike Case study 1, locational marginal prices at different nodes are different due to line congestion. Locational marginal prices of Buses 2, 8 , ad 9 are equal to $24.15 \frac{\$}{\mathrm{MWhr}}$ while this parameter on the other buses of the network is $32.85 \frac{\$}{\mathrm{MWhr}}$, as shown in Fig. 2-a. In this case, generator 3 has to provide more power compared to the previous case since generator 2 cannot transfer more power to supply the load on bus 7 due to line congestion of $L_{78}$. Therefore, power generations of generators 2 is diminished to $135 \mathrm{MW}$ while that of the generator 3 is boosted upto $130 \mathrm{MW}$, as illustrated in Fig. 2-b. The power on the lines are also shown in Fig. 2-c, which obviously depicts that the power flow on the line $L_{78}$ is obviously restricted to the its maximum power limit on both sides $\left(P_{87}=-P_{78}=10 \mathrm{MW}\right)$. As expected, such binding of the line constraint causes an increases in the total cost of the network compared to the case 1 , which is $\$ 5521.38$ (1.7\% higher).
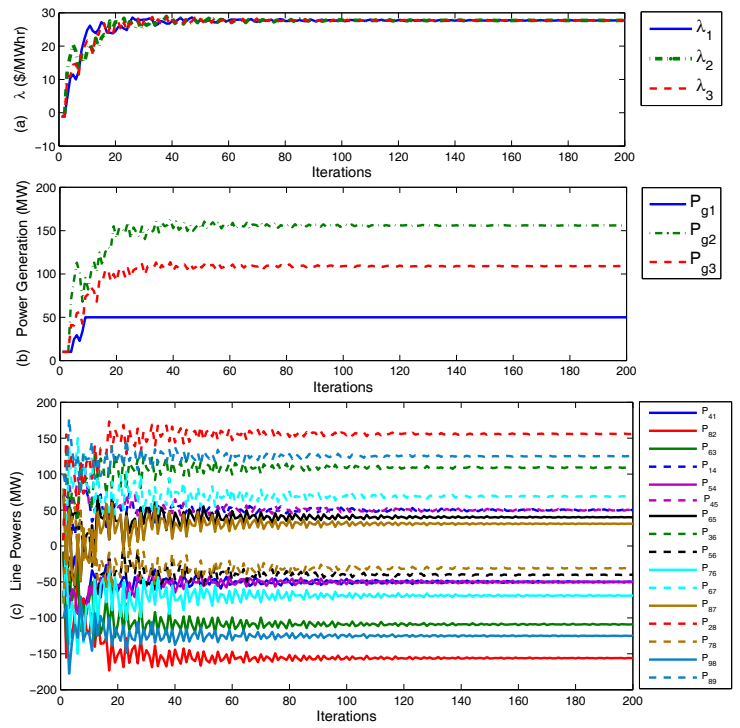

Fig. 1. Simulation results of case 1: (a) Locational Marginal Price (LMP, $\lambda$ ), (b) active power output of generators, (c) power flow on the lines.

\section{CONClusion}

A distributed DC-OPF solution has proposed in this paper based on partial Primal-Dual algorithm. Each bus in the power system is identified as a distinct agent. The algorithm is developed by applying partial Primal-Dual algorithm to economic dispatch problem. The algorithm is finally studied against a radial power network for both congested and noncongested conditions.

\section{REFERENCES}

[1] B. H. Kim and R. Baldick, "Coarse-grained distributed optimal power flow," IEEE Transactions on Power Systems, vol. 12, no. 2, pp. 932-939, 1997.

[2] - "A comparison of distributed optimal power flow algorithms," Power Systems, IEEE Transactions on, vol. 15, no. 2, pp. 599-604, 2000.
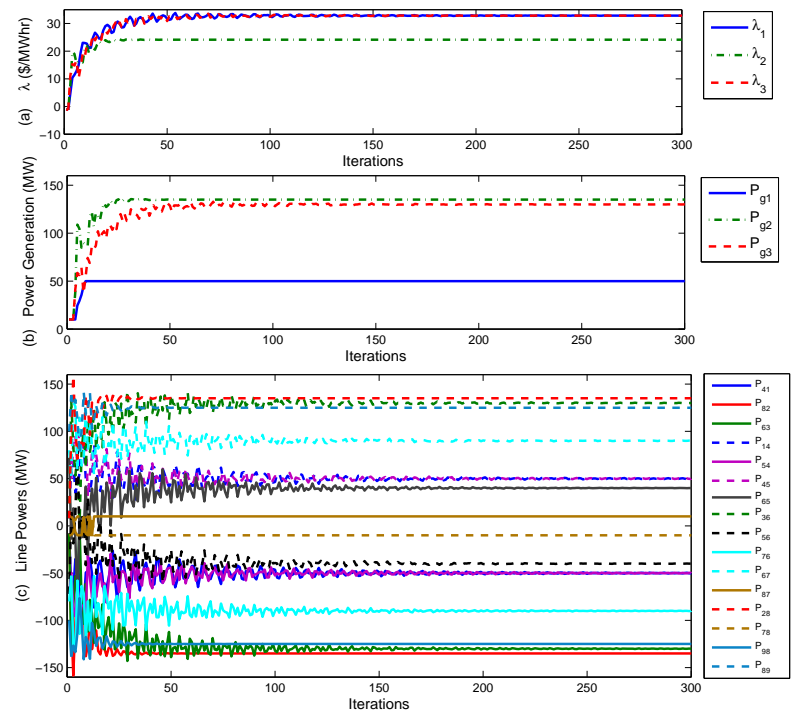

Fig. 2. Simulation results of case 2: (a) Locational Marginal Price (LMP, $\lambda$ ), (b) active power output of generators, (c) power flow on the lines.

[3] T. Erseghe, "A distributed and scalable processing method based upon admm," Signal Processing Letters, IEEE, vol. 19, no. 9, pp. 563-566, 2012.

[4] E. Dall'Anese, H. Zhu, and G. B. Giannakis, "Distributed optimal power flow for smart microgrids," Smart Grid, IEEE Transactions on, vol. 4 no. 3 , pp. $1464-1475,2013$.

[5] V. R. Disfani, L. Fan, L. Piyasinghe, and Z. Miao, "Multi-agent control of community and utility using lagrangian relaxation based dual decomposition," Electric Power Systems Research, vol. 110, pp. 45-54, 2014.

[6] J. Lavaei and S. H. Low, "Zero duality gap in optimal power flow problem," Power Systems, IEEE Transactions on, vol. 27, no. 1, pp. 92-107, 2012.

[7] L. Xiao and S. Boyd, "Optimal scaling of a gradient method for distributed resource allocation," Journal of optimization theory and applications, vol. 129, no. 3, pp. 469-488, 2006.

[8] W. Zhang, W. Liu, X. Wang, L. Liu, and F. Ferrese, "Online optimal generation control based on constrained distributed gradient algorithm," Power Systems, IEEE Transactions on, to appear.

[9] A. J. Conejo and J. A. Aguado, "Multi-area coordinated decentralized dc optimal power flow," Power Systems, IEEE Transactions on, vol. 13, no. 4, pp. 1272-1278, 1998.

[10] A. G. Bakirtzis and P. N. Biskas, "A decentralized solution to the dc-opf of interconnected power systems," Power Systems, IEEE Transactions on, vol. 18, no. 3, pp. 1007-1013, 2003.

[11] P. N. Biskas, A. G. Bakirtzis, N. I. Macheras, and N. K. Pasialis, "A decentralized implementation of dc optimal power flow on a network of computers," Power Systems, IEEE Transactions on, vol. 20, no. 1, pp. 25-33, 2005.

[12] S. Kar, G. Hug, J. Mohammadi, and J. Moura, "Distributed state estimation and energy management in smart grids: A consensus+ innovations approach," Selected Topics in Signal Processing, IEEE Journal of, vol. 8, no. 6, pp. 1022-1038, Dec 2014

[13] A. Chambolle and T. Pock, "A first-order primal-dual algorithm for convex problems with applications to imaging," Journal of Mathematical Imaging and Vision, vol. 40, no. 1, pp. 120-145, 2011.

[14] C. Zhao, U. Topcu, and S. Low, "Swing dynamics as primal-dual algorithm for optimal load control," in Smart Grid Communications (SmartGridComm), 2012 IEEE Third International Conference on. IEEE, 2012, pp. 570-575.

[15] C. Zhao, U. Topcu, N. Li, and S. Low, "Design and stability of load-side primary frequency control in power systems," Automatic Control, IEEE Transactions on, vol. 59, no. 5, pp. 1177-1189, May 2014.

[16] R. D. Zimmerman, C. E. Murillo-Sánchez, and R. J. Thomas, "Matpower: Steady-state operations, planning, and analysis tools for power systems research and education," Power Systems, IEEE Transactions on, vol. 26, no. 1, pp. 12-19, 2011. 